\title{
Das deutsche Gewebegesetz - Anforderungen und Chancen für Gewebebanken und klinische Anwender
}

\author{
Axel Pruß \\ Gewebebank, Institut für Transfusionsmedizin, Charité Universitätsmedizin Berlin, Campus Mitte, Berlin, Deutschland
}

Selten wurde in der letzten Zeit ein Gesetzesentwurf so kontrovers diskutiert, wie die ersten Vorlagen zur Umsetzung der EU-Richtlinie 2004/23/EG über Qualitäts- und Sicherheitsanforderungen für Gewebe und Zellen menschlicher Herkunft. Nach kontrovers geführten Debatten zwischen der Bundesregierung und den zuständigen Bundesbehörden sowie den involvierten medizinischen Fachgesellschaften, Verbänden und Krankenhausträgern wurde das Gesetz über Qualität und Sicherheit von menschlichen Zellen und Geweben (Gewebegesetz) vom 20. Juli 2007 am 1. August 2007 in Kraft gesetzt. Das Gewebegesetz ist ein Artikelgesetz, welches vorhandene Gesetze und Ausführungsbestimmungen, in diesem Fall das Transplantationsgesetz (TPG), das Arzneimittelgesetz (AMG), das Transfusionsgesetz (TFG) sowie die Apothekenbetriebsordnung und die Betriebsverordnung für Arzneimittelgroßhandelsbetriebe verändert.

Von besonderer Bedeutung war der Umstand, dass nunmehr der Entnahme- und der Herstellungsbereich auch im AMG geregelt wurden. Dieser in Deutschland gewählte Weg lehnt sich an die Verfahrensweise bei Blut und Blutprodukten an und garantiert neben einer gesicherten Herstellung in Verbindung mit dem novellierten TPG vor allem Transparenz im Entnahmebereich. Nichtsdestotrotz sind die Anforderungen an die Führung von Gewebebanken, insbesondere Hornhautbanken (die Augenhornhaut war bisher ausschließlich im TPG geregelt), deutlich gestiegen. Grundsätzlich ist jedoch festzuhalten, dass durch diverse Erleichterungen in der Erlaubniserteilung für Entnahme und Herstellung die Weiterführung von Gewebebanken möglich sind. So ist anstatt der bisherigen Herstellungserlaubnis nach $\S 13$ AMG nach Inkrafttreten des Gewebegesetzes eine Erlaubnis nach $\S 20 \mathrm{~b}$ AMG (Entnahme, Testung) und § 20c AMG (Herstellung) für «klassische» Gewebezubereitungen bei der zuständigen Landesbehörde zu beantragen. Die Anforderungen sind gegenüber dem bisherigen §-13-Antrag verringert. Grundsätzlich ent- spricht die Antragsform der des bisherigen §-13-Antrags, jedoch sind die inhaltlichen Anforderungen, z.B. an die Qualifikation der verantwortlichen Person sowie die Be- und Verarbeitungsräume (Gute Fachliche Praxis), vereinfacht worden. Für «klassische» Gewebe und Gewebezubereitungen im Sinne des § 20c AMG (z.B. Knochen, Sehnen, Faszien, Augenhornhaut, Herzklappen, Gefäße, Amnion) wird die Gute Fachliche Praxis entsprechend der Richtlinien 2006/17/EG und 2006/86/ EG in der Arzneimittel- und Wirkstoffherstellungsverordnung (AMWHV) bzw. der TPG-Gewebeverordnung (TPGGewV) umgesetzt. Ergänzend werden Empfehlungen und Richtlinien von Fachkreisen herangezogen, um die Ausführung der AMWHV zu konkretisieren. In diesem Zusammenhang wäre eine Aktualisierung der geweberelevanten Richtlinien des Wissenschaftlichen Beirats der Bundesärztekammer (BÄK) wünschenswert, da die BÄK in der Lage ist, Empfehlungen der Fachkreise zu bündeln und den Anwendern Sicherheit zu geben. Ein erster Schritt hierfür ist die Publikation des Praxisleitfadens «Gewebegesetz» der unter Federführung der BÄK durch kompetente Experten erstellt wurde und Erläuterungen und Hinweise zur Interpretation und Umsetzung des Gewebegesetzes liefert [1]. In diesem Zusammenhang sind auch Aktivitäten verschiedener Fachgesellschaften (z.B. DGCH, DGOOC, DOG, DGTI (Sektion 8 «Gewebezubereitungen»)) zu werten, die mit Ihren Kolloquien, Fachgesprächen bzw. Publikationen zur Weiterentwicklung der Guten Fachlichen Praxis beitragen.

Im vorliegenden Heft ist eine Reihe von wertvollen Beiträgen $\mathrm{zu}$ finden, die einerseits die Interpretation des Gesetzes verdeutlichen und andererseits zu speziellen Fragestellungen der Testung, Entnahme und Herstellung Stellung nehmen. Friedger von Auer [2], Leiter des für den Gesetzestext zuständigen Referats 115 des Bundesministeriums für Gesundheit, erläutert einleitend die Hintergründe der Entwicklung des Gewebegesetzes, die wesentlichen Veränderungen in den novellier-

\section{KARGER}

Fax +497614520714

Information@Karger.de

www.karger.com
() 2008 S. Karger GmbH, Freiburg

Accessible online at:

www.karger.com/tmh
PD Dr. med. Axel Pruß

Gewebebank, Institut für Transfusionsmedizin

Charité - Universitätsmedizin Berlin, Campus Mitte

Charitéplatz 1, 10117 Berlin, Deutschland

Tel. +49 30 450525-161, Fax -976

axel.pruss@charite.de 
ten Gesetzen sowie die Inhalte der resultierenden Verordnungen. Horst Hasskarl und Bita Bahkschai [3] beschäftigen sich in ihrem Beitrag mit der intensiv diskutieren Thematik des Inverkehrbringens von Gewebezubereitungen in Verbindung mit Art und Umfang von Herstellung und Anwendung bzw. mit den $\S \S 4$ (17), 4 (30) und 4a (3) AMG. Die vorgelegte Interpretation hat direkte Auswirkungen auf die Notwendigkeit einer Genehmigung nach § 21a AMG und sollte im Rahmen einer etwaigen Novellierung des AMG berücksichtigt werden. Die Arbeit von Pruß et al. [4] zur Frage der VirusNAT bei Gewebespendern empfiehlt spezielle Herangehensweisen, die auf der Grundlage aller bisher publizierten Virusübertragungen durch Gewebetransplantate sowie der jeweiligen physiologisch-anatomischen Besonderheiten der einzelnen Gewebe basieren. Zu betonen ist, dass die Erarbeitung der Daten sowie die resultierenden Empfehlungen als konzertierte Aktion von führenden deutschen Virologen, Vertretern von Gewebebanken und dem Bereich Virologie des Paul-Ehrlich-Instituts (PEI) entstanden ist. Es ist zu hoffen, dass die Empfehlungen in der Novellierung von Geweberichtlinien der BÄK berücksichtigt werden. Schroeter et al. [5] beschäftigen sich mit dem aktuellen Stand der Entnahme und Bearbeitung von Augenhornhäuten. Von Vorteil war hier, dass die deutschen Hornhautbanken sehr früh die Anforderungen des Gewebegesetzes erkannt und entsprechende Standards erarbeitet haben. Ein Teil der Guten Fachlichen Praxis für Augenhornhäute ist in der vorgelegten Arbeit wiedergegeben. Katthagen et al. [6] beschreiben in Ihrer Übersichtsarbeit die Besonderheiten und speziellen Anforderungen bei der Gewinnung, Prozessierung und Transplantation allogener mus- kuloskelettaler Gewebe. Gelungen ist die kurze Übersicht zum klinischen Einsatz dieser Gewebe, da dieser zumeist nur in orthopädisch-unfallchirurgischen Fachkreisen bekannt ist. Das aus transfusionsmedizinischer Sicht relevanteste Thema bearbeiteten Schlenke et al. [7]. Sie zeigten die besonderen Anforderungen und Konsequenzen des Gewebegesetzes für autologe und allogene hämatopoetische Stammzellpräparationen im Rahmen der aktuellen Gesetze und Verordnungen auf. Der Beitrag von Schilling-Leiß et al. [8] aus der PEI-Arbeitsgruppe um Ralf R. Tönjes wiederum kann als exzellenter Leitfaden und als Verständnishilfe für die Antragsstellung nach $\S 21 \mathrm{a}$ AMG genutzt werden. Schließlich belegen Nitschke et al. [9] auf der Grundlage einer umfassenden Recherche der Zustimmungsraten zur Organ- bzw. Gewebespende in der DSO-Region Nord-Ost, dass eine funktionierende Kooperation zwischen Spendekrankenhäusern, DSO (Deutsche Stiftung Organtransplantation) und Gewebebanken zu exzellenten Zustimmungsraten führen kann. Die Zahlen belegen eindrucksvoll, dass die Gewebespende keinen negativen Einfluss auf die Organspende hat, sondern in geeigneter Form in den Spendeprozess integriert werden kann. Insofern sollten auch andere DSO-Regionen dem Beispiel der DSO-Region NordOst folgen, um dem zunehmenden Mangel an Spendergewebe entgegenzutreten.

Es ist zu hoffen, dass mit dem vorgelegten Heft eine breite Leserschaft für das Thema «Gewebe» interessiert wird und die zweifelsohne vorhandenen Synergieeffekte zwischen Transfusionsmedizinern und Gewebebankern mit weiterem Leben erfüllt werden.

\section{Literatur}

1 Pühler W, Mittel C-D, Hübner M (Hrsg): Praxisleitfaden Gewebegesetz. Grundlagen, Anforderungen, Kommentierungen. Köln, Deutscher Ärzte-Verlag, 2008.

2 Von Auer F: Das Gewebegesetz - Hintergründe und Konsequenzen. Transfus Med Hemother 2008; 35(6):407-413.

3 Hasskarl H, Bakhschai B: Ausgewählte Fragen im Zusammenhang mit dem Gewebegesetz. Transfus Med Hemother 2008;35(6):414-420.

4 Pruß A, Caspari G, Krüger DH, Blümel J, Nübling M, Quenzel E-M, Kalus U, Gerlich W, Gürtler L: Nukleinsäure-Amplifikationstests für HIV, HBV und HCV bei Gewebespendern: sinnvoll oder überflüssig? Transfus Med Hemother 2008;35(6):421430.
5 Schroeter J, Rieck P, Maier P, Reinhard T: Augenhornhaut - Banken und klinische Anwendung. Transfus Med Hemother 2008;35(6):431-437.

6 Katthagen B-D, Scheffler S, Becker R, Willkomm D, Mayr HO, Pruß A: Gewinnung, Prozessierung und Transplantation allogener muskuloskelettaler Gewebe. Transfus Med Hemother 2008;35(6):438445.

7 Schlenke P, Tapernon K, Ahlke C, Mertens A, Sibrowski W: The impact of the German tissue act on the manufacturing of autologous and allogeneic stem cell preparations. Transfus Med Hemother 2008;35(6):446-452.
8 Schilling-Leiß D, Godehardt AW, Scherer J, Cichutek K, Tönjes RR: Genehmigungsverfahren für klassische Gewebezubereitungen gemäß $\S 21 \mathrm{a}$ Arzneimittelgesetz (AMG). Transfus Med Hemother 2008;35(6):453-462.

9 Nitschke FP, Kalus U, Pruß A: Überlegungen zur zukünftigen Umsetzung des Gewebegesetzes im Spendebereich - Erfahrungen bei der Gewebespende und -entnahme im Rahmen der Organspende in der DSO-Region Nord-Ost. Transfus Med Hemother 2008;35(6):464-470. 